\title{
Traces of the XII Aussois Workshop on Combinatorial Optimization
}

\author{
Michael Jünger • Thomas M. Liebling • \\ Denis Naddef • William R. Pulleyblank • \\ Gerhard Reinelt • Giovanni Rinaldi • \\ Laurence A. Wolsey
}

Published online: 9 May 2010

(c) Springer and Mathematical Programming Society 2010

The year 2008, that we declared fiftieth anniversary of integer programming (for it was in 1958 that Ralph Gomory transformed the area with his seminal cutting plane papers), a special edition of the Aussois Workshops in Combinatorial Optimization took place to celebrate that birthday. Many of the founding fathers of the field were present and gave a vivid testimony of those early years, while other well-known

\footnotetext{
M. Jünger $(\bowtie)$

Universität zu Köln, Köln, Germany

e-mail: mjuenger@informatik.Uni-Koeln.DE
}

\section{T. M. Liebling}

EPFL Ecole Polytechnique Fédérale de Lausanne, Lausanne, Switzerland

e-mail: thomas.liebling@epfl.ch

D. Naddef

Grenoble Institute of Technology-Ensimag, Grenoble, France

e-mail: denis.naddef@grenoble-inp.fr

W. R. Pulleyblank

IBM Corporation, Somers, NY, USA

e-mail:wp@us.ibm.com

G. Reinelt

Universität Heidelberg, Heidelberg, Germany

e-mail: gerhard.reinelt@informatik.uni-heidelberg.de

G. Rinaldi

CNR- Istituto di Analisi dei Sistemi, ed Informatica "Antonio Ruberti”, Roma, Italy e-mail: rinaldi@iasi.cnr.it

L. A. Wolsey

Université Catholique de Louvain, CORE, Louvain-la-Neuve, Belgium

e-mail: laurence.wolsey@uclouvain.be 
lecturers gave surveys on some of its most important recent developments. All this is documented in the book [1] Fifty Years of Integer Programming 1958-2008 that came out recently. Further many contributed presentations by leading and junior scientists compellingly illustrated that the field of combinatorial optimization and integer programming more than ever offers exciting challenges that these researchers often turn into breakthroughs. The present issue of MPB contains a selection of those papers, a collection we are particularly pleased with; indeed, some of its items can already be considered as truly pathbreaking. These contributions cover a large spectrum that includes mixed integer, linear and non-linear programming, polyhedral combinatorics, games, etc., ranging from modeling and computational aspects to theory. Here is a brief glimpse of the content of these papers.

"Separation Algorithms for 0-1 Knapsack Polytopes" by Konstantinos Kaparis, and Adam N. Letchford studies valid inequalities for 0-1 knapsack polytopes, in particular separation algorithms for them. It is shown that separation problems for the so-called extended cover and weight inequalities can be solved exactly in $O(n b)$ time and $O\left(\left(n+a_{\max }\right) b\right)$ time, respectively, for $n$ items, knapsack capacity $b$ and largest item weight $a_{\max }$. Fast and effective separation heuristics for the extended cover and lifted cover inequalities are given. Further, an exact separation algorithm for the $0-1$ knapsack polytope itself is given that turns out faster in computation runs than existing methods.

"Strong Valid Inequalities for Orthogonal Disjunctions and Bilinear Covering Sets" by Mohit Tawarmalani, Jean-Philippe Richard, and Kwanghun Chung deals with the problem of finding tight convex envelopes for certain types of nonlinear and mixed-integer nonlinear sets. The specific problems considered are those which consist of the union of a finite number of orthogonal sets.

“Two Row Mixed-Integer Cuts Via Lifting” by Santanu S. Dey, and Laurence A. Wolsey studies the recently characterized extreme valid inequalities of a mixed integer set consisting of two equations with two free integer variables and non-negative continuous variables. It is shown that there exists a unique lifting function that yields extreme inequalities when starting from a maximal lattice-free triangle with multiple integer points in the relative interior of one of its sides, or a maximal latticefree triangle with integral vertices and one integer point in the relative interior of each side. In the other cases (maximal lattice-free triangles with one integer point in the relative interior of each side and non-integral vertices, and maximal lattice-free quadrilaterals), non-unique lifting functions may yield distinct extreme inequalities. For the latter family of triangles, sufficient conditions are given, to yield an extreme inequality for the two row mixed-integer infinite-group problem.

"Convex Relaxations of Non-Convex Mixed Integer Quadratically Constrained Programs: Extended formulations" by Anureet Saxena, Pierre Bonami, and Jon Lee proposes a method to generate strong convex relaxations of Mixed Integer Quadratically Constrained programming problems. The idea is using disjunctive programming and 
lift-and-project to derive cuts from both integrality constraints (not a new issue) and nonconvex quadratic constraints (the contribution of the paper).

"Perspective Reformulations of Mixed Integer Nonlinear Programs with Indicator Variable” by Oktay Günlük, and Jeffrey Linderoth presents a tight formulation for a mixed integer nonlinear set of the form $C_{1} \cup C_{2}$, where $C_{1}$ consists of a single point and $C_{2}$ is a convex set., as well as its representability as a function of the structure of $C_{2}$. They demonstrate computationally the effectiveness of using such a reformulation on certain location, network design and portfolio selection problems.

“Recognizing Binet Matrices" by Antoine Musitelli deals with the problem of determining when a matrix $A$ can be converted to the node-edge matrix of a bidirected graph by elementary row operations. This substantially extends earlier results of a number of several authors determining when $A$ can be converted to the node-edge matrix of a directed graph. Among other consequences, it permits us to determine whether an integer programming problem can be transformed to a b-matching problem on a bidirected graph, in which case it can be solved using Edmonds' and Johnson's b-matching algorithm. The paper presented here describes some of the main ideas of the recognition algorithm which originally appears in the thesis of Musitelli. In particular, it describes the relationship to so-called Camion bases of matrices, introduced by Camion in 1968.

"Max Flow and Min Cut with Bounded-length Paths: Complexity, Algorithms, and Approximation” by A. R. Mahjoub, and S. Thomas McCormick deals with a generalization of maximum flow and minimum cut problems with the additional constraint that flow is restricted to paths having at most $B$ arcs.

“Orientation-based Models for 0,1,2-Survivable Network Design: Theory and Practice”, by Markus Chimani, Maria Kandyba, Ivana Ljubić, and Petra Mutzel presents a new graphical characterization of 2-node connected graphs that leads to two new integer programming formulations, one of which is shown to lead to significant improvements in practice.

"Parametric Nonlinear Discrete Optimization over Well-described Sets and Matroid Intersection” by Yael Berstein, Jon Lee, Shmuel Onn, and Robert Weismantel considers multi-objective nonlinear optimization over discrete finite sets, such as matroids, for which linear optimization is easy. They show that under certain conditions the resulting nonlinear discrete problem is solvable or can be approximated to within a constant factor in polynomial time.

"New Techniques for Cost Sharing in Combinatorial Optimization Games" by Alberto Caprara, and Adam N. Letchford defines a very general class of games, called integer minimization games, which includes the combinatorial optimization games in the literature as special cases. Techniques, based on row and column generation, for computing good cost shares for these games are presented.These techniques are applied on traveling salesman and vehicle routing games. The main 
underlying idea is that suitable valid inequalities for the associated combinatorial optimization problems can be used to derive improved cost shares.

"Efficient and Fair Routing for Mesh Networks" by Andrea Lodi, Enrico Malaguti, and Nicolás E. Stier-Moses was inspired by the One Laptop Per Child project; it considers mesh networks that connect devices that cannot recharge their batteries easily. It studies how the mesh should retransmit information to make use of the energy stored at the nodes effectively. A distributed and online routing algorithm is compared to an offline, centralized approach. The centralized approach, besides being unrealistic in terms of information requirements, is also NP-hard to solve. Therefore, attention is focused on the former; extensive computational experiments are conducted to evaluate the efficiency and fairness achieved with it.

"Solving a Real-World Train-Unit Assignment Problem" by Valentina Cacchiani, Alberto Caprara, and Paolo Toth concerns an application of integer programming to a practically relevant railway scheduling problem. The problem is to assign train units to timetabled trips such that the passenger demand is satisfied and that the train lengths respect some upper bounds. Additional real life requirements such as maintenance and deadheading are also taken into account.

"Locating Leak Detecting Sensors in a Water Distribution Network by Solving Prize-Collecting Steiner Arborescence Problems" by Alain Prodon, Scott DeNegre, and Thomas M. Liebling presents the practical problem of locating acoustic leak sensors in a water distribution network. The problem is formulated as a prize-collecting Steiner problem and new Branch-and-Cut-and-Bound approaches to solve such problems are compared with existing ones. In particular the approach is used to solve exactly real world problems for cities the size of Lausanne.

"The first cut is the cheapest: copositivity improves SDP bounds for the clique number" by Immanuel M. Bomze, Florian Frommlet, and Marco Locatelli explores a generic cutting-plane approach to strengthen the Lovász-Schrijver semidefinite programming bound on the clique number of a graph by adding linear cuts based on copositive matrices.

"On the Dominant of the s-t-cut Polytope" by Martin Skutella, and Alexia Weber gives a complete description of the dominant of the s-t cut polytope, by characterizing its vertices, facets, and adjacency of vertices, for both directed and undirected graphs.

"Separation, Dimension, and Facet Algorithms for Node Flow Polyhedra" by Meret Martens, S. Thomas McCormick, and Maurice Queyranne is motivated by applications to production planning, the paper presents an algorithm to solve the facet separation problem for the node flow polyhedron by developing algorithms for solving the separation, violation, and face dimension subproblems. 
"Mixed-integer Sets from two Rows of two Adjacent Simplex Bases" by Ken Andersen, Quentin Louveaux, and Robert Weismantel studies the structure and geometry of a mixed-integer set obtained from considering two rows of a simplex tableau, where some of the non-basic variables have a finite upper bound. The results are very interesting for generating strong cutting planes from multiple rows of LP simplex tableaux.

"Small Chvátal Rank" by Tristam Bogart, Annie Raymond, and Rekha Thomas describes a variant of the Chvátal-Gomory procedure that will produce a sufficient set of facet normals for the integer hulls of all polyhedra $\{x: A x \leq b\}$ as $b$ varies. The authors introduce a vector version of the Chvátal-Gomory procedure called Iterated Basis Normalization (IBN) and define the Small Chvátal Rank to be the number of rounds of IBN required. They characterize problems having Small Chvátal Rank equal to zero, develop other properties and show how the theory applies to the stable set problem in graphs.

"Computable Representations for Convex Hulls of Low-dimensional Quadratic Forms" by Kurt Anstreicher, and Samuel Burer deals with the convex hull of the matrix representations of products of variables over two important types of feasible sets, the simplex and the box.

“Speeding up IP-based Algorithms for Constrained Quadratic 0-1 Optimization" by Christoph Buchheim, Frauke Liers, and Marcus Oswald presents a general framework for solving constrained quadratic 0-1 problems and reports on computational experiments for instances of the matching, the linear ordering, and the assignment problem with quadratic objective function.

"Approximating a Class of Combinatorial Problems with a Rational Objective Function" by José Correa, Cristina Fernandes, and Yoshiko Wakabayashi shows that, if there is an $\alpha$-approximation for the problem of minimizing a nonnegative linear function subject to constraints satisfying a certain increasing property, then there is an $\alpha$-approximation ( $1 / \alpha$-approximation) for the problem of minimizing (maximizing) a nonnegative rational function subject to the same constraints. The framework applies in particular to covering problems and network design problems. It extends a 1979 result of Megiddo in a different way from a 1987 result of Hashizume et al.

"A Note on Separation from Membership and Folklore" by Jean-François Maurras" shows that separation from membership for well defined non empty polyhedra can be considered to lie in the folklore of mathematical programming.

"A Note on the Selection of Benders' cuts" by Matteo Fischetti, Domenico Salvagnin, and Arrigo Zanette proposes some modifications to Benders' cut generation aimed at improving the practical performance of a cutting plane algorithm based on these cuts. 
Here we would like to thank MPB EIC Danny Ralph for having accepted to host this special issue, and helped us carry the project to a happy end. We also thank Marianne Ruegg for her precious support and last but not least, we are indebted to all referees, who will have to remain anonymous, for the very competent and careful work to assure the quality and relevance of the papers included in this issue.

Cologne, Lausanne, Grenoble, New York, Heidelberg, Rome, Louvain-la-Neuve, March 2010

\section{Reference}

1. Jünger, M., Liebling, Th.M., Naddef, D., Nemhauser, G.L., Pulleyblank, W.R., Reinelt, G., Rinaldi, G., Wolsey, L.A. (eds.): 50 Years of Integer Programming 1958-2008, From the Early Years to the Stateof-the-Art, 804 with 2 DVD. Springer, Heidelberg (2010) 